\title{
Vorwort zur 1. Auflage
}

Dieses Buch will den gesamten Prozess qualitativer Forschung begleiten - von den ersten Anfängen bis zur Niederschrift der Ergebnisse. Es soll bei den Entscheidungen, die im Verlauf dieses Prozesses zu treffen sind, gleichermaßen praktische Hilfestellung geben wie auch dabei helfen, diese Entscheidungen zu reflektieren. Wie praktiziere ich einen bestimmten Schritt im Forschungsprozess? Und was tue ich und mit welchen Konsequenzen, wenn ich mich für dieses Vorgehen anstelle eines anderen entscheide?

Auch wenn das Feld der qualitativen oder rekonstruktiven Sozialforschung traditionell in verschiedene Schulen aufgeteilt ist, geht dieses Buch von Fragen aus, die sich alle diese Schulen gleichermaßen stellen müssen; Fragen überdies, die sie an vielen Stellen mit dem anderen großen „Lager“, der standardisierten Sozialforschung, verbinden. Wie formuliert man sein Erkenntnisinteresse und seine Fragestellung? Welche methodischen Zugänge sind dem angemessen? Wo findet man dafür die adäquaten Daten? Wie erschließt man ein Forschungsfeld? Wie dokumentiert und interpretiert man empirisches Material? Wofür stehen die empirischen Fälle und die daraus gewonnenen Befunde? Wie kommt man zur Formulierung einer Theorie? Und wie schreibt man das Ergebnis des Forschungsprozesses so nieder, dass auch andere Lust bekommen, darüber etwas zu lesen, und gleichzeitig etwas davon erfahren, wie dieses Ergebnis zustande gekommen ist?

Der Aufbau des Buches orientiert sich in erster Linie am Prozess der Forschung und zeichnet einen kompletten Forschungsbogen nach. Erst in zweiter Linie kommen verschiedene Schulen und Ansätze im Bereich der interpretativen Sozialforschung zu Wort. Aus diesen Schulen mussten wir hier eine Auswahl treffen, und es gibt zweifellos weitere wichtige Richtungen, die man hätte behandeln können. Dennoch glauben wir, mit der Auswahl der Ansätze ein Spektrum zu repräsentieren, in dem sich die wesentlichen Pole des Feldes qualitativer Sozialforschung, aber auch die zentralen gemeinsamen Problemstellungen wiederfinden. Gemeinsam allerdings ist allen hier behandelten Ansätzen, dass sie bei der Interpretation stets auf zwei Ebenen ansetzen und diese zueinander ins Verhältnis setzen: Dass sie nämlich den Inhalt ins Verhältnis setzen zur interaktiven Form, in der er präsentiert wird. Und dass sie beides über den systematischen und methodisch angeleiteten Vergleich erschließen. Wenn es in diesem Buch ein Credo gibt, dann ist es das: Qualitative Forschung besteht nicht darin, zu paraphrasieren, nachzuzeichnen und zu klassifizieren, sondern sie soll in methodisch begründeter Weise zu anspruchsvollen Interpretationen und - über das Nutzen des systematischen Vergleichs - zu begründeten Generalisierungen und zur Theorie gelangen.

Ohne die Divergenzen zwischen den verschiedenen hier behandelten Ansätzen unter den Tisch fallen zu lassen, wollen wir diese doch in einer Weise präsentieren, in der sie sich wechselseitig nicht grundsätzlich ausschließen. Auch wenn es unter- 
schiedliche theoretische Grundlegungen gibt, die man kennen sollte, stößt man doch in der Forschungspraxis immer wieder auf Gemeinsames. Das gilt auch für uns als Autorinnen dieses Buches, die wir aus unterschiedlichen methodischen Richtungen und zudem aus unterschiedlichen fachlichen Disziplinen kommen. Das Schreiben dieses Buches war für uns stets aufs Neue ein Prozess wechselseitiger Perspektivenübernahme. Es war aber auch immer wieder ein Prozess der (wechselseitig aneinander herangetragenen) Kritik an manch unhinterfragter Selbstverständlichkeit methodischer Schulen, die sich bisweilen nur noch nach innen, aber nicht mehr nach außen verständlich machen. Die Arbeit am konkreten Material jedoch war immer gemeinsames Interpretieren, bei dem Schulendivergenzen nachrangig wurden.

Die Geschichte des Schreibens an diesem Buch reicht buchstäblich zurück in die Jahre des letzten Jahrtausends. Seitdem kamen Kinder zur Welt, wurden Umzüge bewältigt, neue Stellen angetreten und viele andere Projekte begonnen und abgeschlossen. Das Buch hat uns ständig begleitet, und manchmal waren wir nahe daran, es ad acta zu legen. Der normale Wissenschafts- und Lehrbetrieb trägt das Seine dazu bei, dass solche größeren Vorhaben sehr mühsam werden. Es war nicht zuletzt ein Forschungssemester am Europäischen Hochschulinstitut in Florenz, das die Fertigstellung des Buches nun endlich möglich gemacht hat. Den Lektoren des Oldenbourg Verlages - Arno Mohr und Jürgen Schechler - sei für den langen Atem bei der Betreuung dieses Projektes gedankt. Bei der konkreten Fertigstellung und Edierung des Manuskripts waren Verena Hauser (Wien) und Melanie Sachs (Leipzig) eine unschätzbare Hilfe.

Die lange Dauer des Schreibens an diesem Buch hatte aber ohne Zweifel einen großen Vorteil: In dieser Zeit waren wir mit den empirischen Projekten und methodischen Fragen Hunderter Studierender - in Vorlesungen, Seminaren und Forschungswerkstätten - konfrontiert, wurden von ihnen angeregt und angefragt und mussten mit ihnen gemeinsam Lösungen erarbeiten. So mühsam das bisweilen war (und so gerne wir dabei schon dieses Buch zur Hand gehabt hätten), haben wir davon doch auch außerordentlich profitiert. Wir hoffen, dass dies auch den Studierenden zugutekommt, die diese kondensierten Erfahrungen nun als Lehrbuch in die Hand nehmen.

Wien und Florenz im Februar 2008

Aglaja Przyborski und Monika Wohlrab-Sahr 